\title{
Risk Evaluation in Failure Mode and Effects Analysis Based on D Numbers Theory
}

\author{
B. Liu, Y. Deng
}

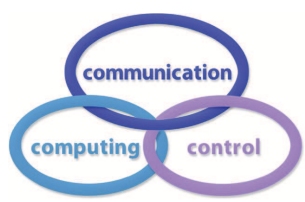

\section{Baoyu Liu}

1. Institute of Fundamental and Frontier Science

University of Electronic Science and Technology of China

Chengdu, 610054, China

2. School of Data and Computer Science

Sun Yat-sen University, China

Guangzhou, China

Baoyu.L@hotmail.com

\section{Yong Deng*}

Institute of Fundamental and Frontier Science

University of Electronic Science and Technology of China

Chengdu, 610054, China

*Corresponding author: prof.deng@hotmail.com

\begin{abstract}
:
Failure mode and effects analysis (FMEA) is a useful technology for identifying the potential faults or errors in system, and simultaneously preventing them from occurring. In FMEA, risk evaluation is a vital procedure. Many methods are proposed to address this issue but they have some deficiencies, such as the complex calculation and two adjacent evaluation ratings being considered to be mutually exclusive. Aiming at these problems, in this paper, A novel method to risk evaluation based on D numbers theory is proposed. In the proposed method, for one thing, the assessments of each failure mode are aggregated through D numbers theory. For another, the combination usage of risk priority number (RPN) and the risk coefficient newly defined not only achieve less computation complexity compared with other methods, but also overcome the shortcomings of classical RPN. Furthermore, a numerical example is illustrated to demonstrate the effectiveness and superiority of the proposed method.
\end{abstract}

Keywords: failure mode and effects analysis, Dempster-Shafer evidence theory, D numbers, risk evaluation, aggregate assessment.

\section{Introduction}

Failure mode and effects analysis (FMEA) is an efficient technology for identifying potential faults, problems, risk, and errors from the system, procedure, and service. It improves the reliability by preventing these faults, problems, risks, and errors from occurring. Risk evaluation is a pivotal procedure in FMEA. Nowadays, FMEA is developed so fast that it is extensively applied in many fields, such as medical care [3,29,32,49,60], society [51], environmental protection $[1,8]$, financial service [65], industry $[2,24,69,78]$, and so on.

A traditional method for risk evaluation in FMEA is the classical risk priority number (RPN), which is obtained by multiplying the grades of the occurrence assessment, severity assessment, and detection assessment. Therefore, how to aggregate the assessment information of 
these three risk factors is a significant issue, especially when it comes to the information with uncertainty. Focusing on this problem, many math models such as fuzzy sets $[14,16,17,79], \mathrm{R}$ numbers [53], D numbers [20], Z numbers [21,26,27,39,76] and evidence theory [10,55], have been applied to the real applications $[37,50]$. In [28], Kim et al. present a general model to explain the functional relationship among the three factors, and use the model to discuss the unique role of each factor for comparing the risk of different failure modes. In [77], a new method to risk evaluation based on Dempster-Shafer evidence theory is proposed. Some other evidential FEMA are presented recently [5].

Nevertheless, although classical RPN is easy to use because of its succinct form, it is still criticized for its weaknesses. For example, the experts usually give the assessment with uncertainty or fuzzy information, but classical RPN is not appropriated to treat the fuzzy assessment. Furthermore, different combination of risk factors might acquire the same RPN, however, the potential risk might be totally different so that they might have different priorities. With the aim of overcoming these weaknesses, many methods, such as Chin's method [9], are proposed. However, existing methods almost is not only too complex to calculation, but also do not take the non-exclusiveness between two adjacent rankings into account. Actually, because of the subjectivity of the experts, two adjacent estimation scales are supposed to be not exclusive mutually. In order to solve the problems, a novel method based on D number theory [13] is proposed in this paper. On the one hand, the assessments for each failure mode are aggregated through constructing and combining D numbers because two propositions are allowed to be non-exclusive in D numbers theory. On the other hand, RPN is applied in the proposed method so as to reduce the computation complexity, simultaneously, novel compute mode to RPN and risk coefficient present in this paper is capable to get rid of the weaknesses of the classical RPN. Last but not the least, an illustrative example is used to show the effectiveness and superiority of the proposed method.

The remainder of this paper is organized as follows. Key concepts and previous theories are reviewed in short in Section 2. In Section 3, A novel risk evaluation in failure mode and effects analysis based on D numbers theory is proposed. To demonstrate the effectiveness and superiority of the proposed method, a numerical example is illustrated in Section 4. Last but not the least, A brief conclusion is drawn in Section 5.

\section{Preliminaries}

\subsection{Dempster-Shafer evidence theory}

The real application is inevitable to deal with uncertainty [4,6, 45-47]. Dempster-Shafer evidence theory (D-S theory) $[10,55]$, is a significant theory to handle uncertainty information [11]. Compared with Bayesian theory, it needs weaker conditions so that it is often deemed as an extension of the Bayesian theory. D-S theory is widely applicated in many fields, such as decision making [6,22,30,44,71], pattern recognition [40,41,43,73], evidential reasoning [15, 42,74, 83-85], risk and reliability $[52,54]$, information fusion $[59,61,75]$, uncertainty modelling $[19,25,58]$ and conflict management $[36,68,81]$.

Definition 1. Let $\Theta=\left\{H_{1}, H_{2}, \cdots, H_{N}\right\}$ be a finite nonempty set, which consist of $\mathrm{N}$ mutually exclusive elements. Let $P(\Theta)$ be the power set of $\Theta$, which is composed of $2^{N}$ elements. The basic probability assignment (BPA) function is defined as a mapping of the power set $P(\Theta)$ to a number between 0 to 1 , that is $m: P(\Theta) \rightarrow[0,1]$, and which satisfies the following conditions:

$$
m(\emptyset)=0
$$




$$
\sum_{A \in P(\Theta)} m(A)=1 .
$$

The mass $\mathrm{m}(\mathrm{A})$ represents how strongly the evidence supports to A.

Definition 2. Let $m_{1}, m_{2}$ be two BPAs defined on the frame of discernment $\Theta$. The Dempster's combination rule, denoted by $m=m_{1} \bigoplus m_{2}$, is defined as follows:

$$
m(A)= \begin{cases}\frac{1}{k-1} \sum_{B \cap C=A} m_{1}(B) m_{2}(C), & A \neq \emptyset \\ 0, & A=\emptyset\end{cases}
$$

with

$$
K=\sum_{B \cap C=\emptyset} m_{1}(B) m_{2}(C)
$$

where $\mathrm{K}$ is a normalization constant which reflects the conflict of two bodies of evidence.

Actually, $0 \leq K \leq 1$. $K=0$ shows the absence of conflict between two bodies of evidence. While $K=1$ shows complete conflict between $m_{1}$ and $m_{2}$. Besides, when $K=1$, the Dempster's combination rule is not any longer applicable, a possible explanation is open world assumption $[32,33]$. In order to make decision in terms of the BPA, a method called pignistic probability transformation is present in [57], which derive a distribution of probabilities from the BPA. The pignistic probability transformation function is defined as follows:

Definition 3. Let $m$ be a BPA on the frame of discernment $\Theta$, a pignistic probability transformation function $\operatorname{Bet}_{m}: \Theta \longrightarrow[0,1]$ associated to $m$ is defined by

$$
\operatorname{Bet}_{m}(x)=\sum_{x \in A, A \in \Theta} \frac{1}{|A|} \frac{m(A)}{1-m(\emptyset)}
$$

where $m(\emptyset) \neq 1$ and $|A|$ is the cardinality of proposition $A$.

\section{$2.2 \quad$ Fuzzy set theory}

Fuzzy sets were proposed independently by Zadeh [79] in 1965 as an extension of the classical notion of set. Fuzzy set theory is widely applied in many fields $[23,33,72]$. It reflects the stay of the object and its fuzzy concept as a fuzzy set. Then, it sets up the appropriate membership functions through fuzzy set about operation and transform, and analyzes the fuzzy object based on the fuzzy mathematics. In the objective world, there are many fuzzy phenomena. For example, when evaluating a person's appearance, people usually use linguistic variables whose values are represented by words or sentences in a natural or artificial language, such as "very pretty", "pretty", "general", "ugly", and "very ugly".

Definition 4. Denote $L$ as the universe of discourse, a fuzzy set $A$ is described by a membership function $\mu_{A}$ satisfying

$$
\mu_{A}: L \longrightarrow[0,1]
$$

where $\mu_{A}(x)$ is called the membership degree of $x \in L$ belonging to fuzzy set $A$.

For $L=\left\{x_{1}, \ldots, x_{i}, \ldots, x_{n}\right\}$, the fuzzy set $\left(A, \mu_{A}\right)$ is represent by

$$
\frac{\mu_{A}\left(x_{1}\right)}{x_{1}}, \ldots, \frac{\mu_{A}\left(x_{i}\right)}{x_{i}}, \ldots, \frac{\mu_{A}\left(x_{n}\right)}{x_{n}} .
$$




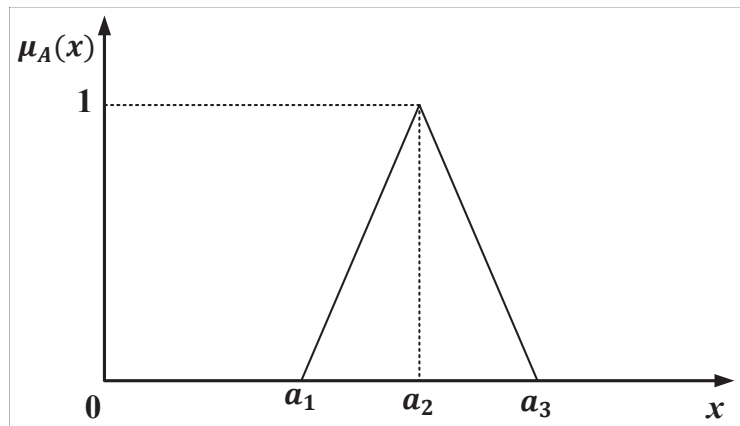

Figure 1: Graphical presentation of the triangular fuzzy number

It is obvious that a fuzzy set is characterized entirely by its membership function. When $\mu_{A}(x)$ get value from $\{0,1\}$, fuzzy set $A$ degrade into a classical set. $A$ is a fuzzy subset of the real number $\mathrm{R}$, and its membership function satisfies

$$
\mu_{A}(x): R \longrightarrow[0,1]
$$

where $x$ is real number and there exists an element $x_{0}$ such that $\mu_{A}=1$. Triangular fuzzy numbers are the most extensively applied fuzzy numbers. A triangular fuzzy number is usually expressed as $A=\left(a_{1}, a_{2}, a_{3}\right)$, as graphically shown in Figure 1, which has the following membership function

$$
\mu_{A}(x)= \begin{cases}0, & x<a_{1} \\ \frac{x-a_{1}}{a_{2}-a_{1}}, & a 1 \leq x \leq a 2 \\ \frac{a 3-x}{a 3-a 2}, & a_{2} \leq x \leq a_{3} \\ 0, & x>a_{3}\end{cases}
$$

where $a_{1}<a_{2}<a_{3}$.

In practice, fuzzy numbers are bound up with linguistic variables to describe the fuzzy evolution to objects.

\subsection{Risk priority number}

The real systems are too complicated to be modelled. Risk priority number (RPN) is a traditional and typical method to model and evaluate risk in FMEA. RPN is calculated by multiplying the grades of occurrence assessment (O), severity assessment (S), and detection assessment (D). That is

$$
\mathrm{RPN}=\mathrm{O} \times \mathrm{S} \times \mathrm{D}
$$

where $\mathrm{O}$ stands for the probability of occurrence of failure mode, $\mathrm{S}$ refers to the severity of failure mode and D refers to the probability of failure being detected. The three risk factors are evaluated by FMEA experts using a 1 to 10 numeric scale. Besides, occurrence assessment is expressed in Table 1. The larger RPN is, the more important degree it is supposed to be assigned, referring to the failure mode should be more priority to be corrected. Although this method is easy to use because of its sententious form, traditional RPN is still criticized for its weaknesses. For example, traditional RPN does not take the weights of three risk factors into consideration. Besides, different combination of risk factors might acquire the same RPN, however, the potential risk might be totally different so that they might have different priorities. 


\subsection{D numbers}

D number, is a useful tool to model uncertain information, which overcomes the shortcomings of Dempster-Sharfer theory [20]. Nowadays, D number is widely used in many fields such as decision making $[7,19,31]$, risk assessment $[18,29,35,67]$, reliability analysis $[70,80]$, data fusion $[8,62,66]$. It is defined as follows:

Definition 5 . Let $\Omega$ be a finite nonempty set, D number is a mapping $D: \Omega \longrightarrow[0,1]$, such that

$$
\sum_{B \subseteq \Omega} D(B) \leq 1 \text { and } D(\emptyset)=0
$$

where $\emptyset$ is the empty set and $B$ is a subset of $\Omega$.

If $D(B)=1$, the information is regarded to be complete while $D(B)<1$, it is considered to be incomplete. Most importantly, different from D-S theory, D number does not request the elements of set $\Omega$ to be mutually exclusive. In order to express the non-exclusiveness in $\Omega$, a fuzzy membership function is used to measure the exclusive/non-exclusive degree [82].

Definition 6. Let $A_{i}$ and $A_{j}$ be two non-empty elements in $2^{\Omega}$, the non-exclusive degree between $A_{i}$ and $A_{j}$ is characterized by a fuzzy membership function $u_{\neg E}$ as follows:

$$
u_{\neg E}: 2^{\Omega} \times 2^{\Omega} \longrightarrow[0,1]
$$

with

$$
u_{\neg E}\left(A_{i}, A_{j}\right)= \begin{cases}1, & A_{i} \cap A_{j} \neq \emptyset \\ p, & p \in[0,1], A_{i} \cap A_{j}=\emptyset\end{cases}
$$

besides,

$$
u_{\neg E}\left(A_{i}, A_{j}\right)=\max _{x \in A_{i}, y \in A_{j}}\left\{u_{\neg E}(x, y)\right\}
$$

Let $u_{E}$ be the exclusive degree between $A_{i}$ and $A_{j}$, then $u_{E}=1-u_{\neg E}$.

An illustrative example is given as follows to express the calculation of the non-exclusive degree.

Table 1: Assessment rankings for occurrence in FMEA

\begin{tabular}{lll}
\hline Ranking & Probability of Occurrence & Possible Failure Rate \\
\hline 10 & Extremely high: failure almost inevitable & $\geq 1 / 2$ \\
9 & Very high & $1 / 3$ \\
8 & Repeated failures & $1 / 8$ \\
7 & High & $1 / 20$ \\
6 & Moderately high & $1 / 80$ \\
5 & Moderate & $1 / 400$ \\
4 & Relatively low & $1 / 2000$ \\
3 & Low & $1 / 15000$ \\
2 & Remote & $1 / 150000$ \\
1 & Nearly impossible & $\leq 1 / 150000$ \\
\hline
\end{tabular}






Figure 2: Graphically presentation of fuzzy variables in Table 2

Example 7. Suppose there is a non-empty set $\Omega=\{a, b, c\}$, where $a, b, c$ are three fuzzy variables represented by triangular fuzzy numbers given in Table 2 and shown in Figure 2. The nonexclusiveness degree can be calculated as follows [12]:

$$
u_{\neg E}(A, B)=\frac{\text { Area }_{A \cap B}}{\text { Area }_{A}+\text { Area }_{B}-\text { Area }_{A \cap B}}
$$

where the areas of fuzzy numbers $A$ and $B$ are characterized by $A r e a_{A}$ and $A r e a_{B}$, and the area of the overlap of $A$ and $B$ is Area $a_{A \cap B}$. Thus, according to Eq.12, we have

$$
\begin{aligned}
u_{\neg E}(a, b) & =\frac{\text { Area }_{a \cap b}}{\text { Area }_{a}+\text { Area }_{b}-\text { Area }_{a \cap b}}=\frac{0.25}{1+1-0.25} \approx 0.1429 \\
u_{\neg E}(b, c) & =\frac{\text { Area }_{b \cap c}}{\text { Area }_{b}+\text { Area }_{c}-\text { Area }_{b \cap c}}=\frac{0.25}{1+1-0.25} \approx 0.1429 \\
u_{\neg E}(a, b, c) & =\max \left\{u_{\neg E}(a, b), u_{\neg E}(b, c)\right\}=\max \{0.1429,0.1429\}=0.1429 \\
u_{\neg E}(a,\{a, b\}) & =\max \left\{u_{\neg E}(a, a), u_{\neg E}(a, b)\right\}=\max \{1,0.1429\}=1
\end{aligned}
$$

Similar with Dempster combination rule, the combination rule is discussed under two cases: complete information and incomplete information.

Definition 8. (complete information). Let $D_{1}$ and $D_{2}$ be two numbers over $\Omega$ with $\sum_{A \subseteq \Omega} D_{1}(A)=1$ and $\sum_{A \subseteq \Omega} D_{2}(A)=1$, the combination of $D_{1}$ and $D_{2}$, indicated by $D=D_{1} \odot D_{2}$, is defined by

$$
D(A)= \begin{cases}0, & A=\emptyset \\ \frac{1}{1-K_{D}}\left(\sum_{B \cap C=A} u_{\neg E}(B, C) D_{1}(B) D_{2}(C)+\right. & \\ \left.\sum_{B \cup C=A, B \cap C=\emptyset} u_{\neg E}(B, C) D_{1}(B) D_{2}(C)\right), & A \neq \emptyset\end{cases}
$$

with

$$
K_{D}=\sum_{B \cap C=\emptyset}\left(1-u_{\neg E}(B, C)\right) D_{1}(B) D_{2}(C)
$$

Table 2: The fuzzy number of fuzzy variables

\begin{tabular}{cc}
\hline Fuzzy Variables & Fuzzy Numbers \\
\hline $\mathrm{a}$ & $(1,2,3)$ \\
$\mathrm{b}$ & $(2,3,4)$ \\
$\mathrm{c}$ & $(3,4,5)$ \\
\hline
\end{tabular}


It is worth mentioning that this combination rule can be degenerated to the classical Dempster's rule if $u_{\neg E}=0$ for any $B \cap C=\emptyset$. a numerical example is used to illustrate the combination of two D numbers under the complete information situation as follows:

Example 9. There are two $D$ numbers over $\Omega=\{a, b\}$ :

$$
\begin{array}{ll}
D_{1}(a)=0.4, & D_{1}(b)=0.6 \\
D_{2}(a)=0.9, & D_{2}(b)=0.1
\end{array}
$$

And assume $u_{\neg E}(a, b)=0.1429$. Thus, we have $D \prime=D_{1} \odot D_{2}$ that

$$
\begin{aligned}
K_{D^{\prime}} & =(1-0.1429) \times[0.4 \times 0.1+0.6 \times 0.9]=0.4971 \\
D^{\prime}(a) & =\frac{1}{1-0.4971} \times 1 \times 0.4 \times 0.9=0.7159 \\
D^{\prime}(b) & =\frac{1}{1-0.4971} \times 1 \times 0.6 \times 0.1=0.1193 \\
D^{\prime}(a, b) & =\frac{1}{1-0.4971} \times 0.1429 \times[0.4 \times 0.1+0.6 \times 0.9]=0.1648
\end{aligned}
$$

Definition 10. (incomplete information). Let $D_{1}$ and $D_{2}$ be two numbers over $\Omega$ with $\sum_{A \subseteq \Omega} D_{1}(A)<1$ and $\sum_{A \subseteq \Omega} D_{2}(A)<1$, the combination of $D_{1}$ and $D_{2}$, defined by $D=D_{1} \odot D_{2}$ and calculated as follows:

$$
D(A)= \begin{cases}0, & A=\emptyset \\ f\left(Q_{1}, Q_{2}\right) \frac{D_{t}(A)}{\sum_{B \subseteq \Omega} D_{t}(B)}, & A \neq \emptyset\end{cases}
$$

with

$$
D_{t}(A)=\sum_{B \cap C=A} u_{\neg E}(B, C) D_{1}(B) D_{2}(C)+\sum_{B \cup C=A, B \cap C=\emptyset} u_{\neg E}(B, C) D_{1}(B) D_{2}(C), \forall A \in \Omega
$$

and

$$
Q_{1}=\sum_{A \subseteq \Omega} D_{1}(A), \quad Q_{2}=\sum_{A \subseteq \Omega} D_{2}(A)
$$

where $f\left(Q_{1}, Q_{2}\right)$ is a function satisfying $0 \leq f\left(Q_{1}, Q_{2}\right) \leq \max \left\{Q_{1}, Q_{2}\right\}, f\left(Q_{1}, Q_{2}\right)=1$ if $Q_{1}=1$ and $Q_{2}=1$.

Next, a simple example is used to illustrate the combination process of D numbers according to Definition 10.

Example 11. There are two $D$ numbers over $\Omega=\{a, b\}$ :

$$
\begin{array}{ll}
D_{1}(a)=0.7, & D_{1}(b)=0.2 \\
D_{2}(a)=0.5, & D_{2}(b)=0.3
\end{array}
$$


Assume $u_{\neg E}(a, b)=0.1429$, and let $f\left(Q_{1}, Q_{2}\right)=Q_{1} \times Q_{2}$. Thus, we have $D=D_{1} \odot D_{2}$ that

$$
\begin{aligned}
f\left(Q_{1}, Q_{2}\right) & =(0.7+0.2) \times(0.5+0.3)=0.72 \\
D_{t}(a) & =0.7 \times 0.5=0.35 \\
D_{t}(b) & =0.2 \times 0.3=0.06 \\
D_{t}(a, b) & =0.1429 \times(0.7 \times 0.3+0.2 \times 0.5) \approx 0.044
\end{aligned}
$$

Thus,

$$
\begin{gathered}
\sum_{B \subseteq \Omega} D_{1}(B)=0.35+0.06+0.044=0.454 \\
D(a)=f\left(Q_{1}, Q_{2}\right) \frac{D_{t}(a)}{\sum_{B \subseteq \Omega} D_{1}(B)} \approx 0.555 \\
D(b)=f\left(Q_{1}, Q_{2}\right) \frac{D_{t}(b)}{\sum_{B \subseteq \Omega} D_{1}(B)} \approx 0.095 \\
D(a, b)=f\left(Q_{1}, Q_{2}\right) \frac{D_{t}(a, b)}{\sum_{B \subseteq \Omega} D_{1}(B)} \approx 0.070
\end{gathered}
$$

If there are $n \mathrm{D}$ numbers expressed as $D_{1}, D_{2}, \cdots, D_{n}$, whose weights are $w_{1}, w_{2}, \cdots, w_{n}$, satisfying $\sum_{i=1}^{n} w_{i}=1$. At first, the average $\mathrm{D}$ number among $D_{1}, D_{2}, \cdots, D_{n}$ is defined as

$$
\bar{D}(A)=\sum_{i=1}^{n} w_{i} D_{i}(A), \forall A \subseteq \Omega
$$

Then, the result of combination $D_{1}, D_{2}, \cdots, D_{n}$ is acquired by combining average D number $\bar{D}$ with itself $n-1$ times.

$$
D=\bar{D} \bigodot \bar{D} \bigodot \cdots \bigodot \bar{D}
$$

where $\odot$ is the combination rule given in Definition 8 and Definition 10.

\section{Proposed method}

Failure mode and analysis (FMEA) is an efficient technology to identify and remove potential faults, errors and risk from systems. In FMEA, risk evaluation is a significant procession. Traditionally, risk priority number (RPR) is used to evaluate risk, which is calculated by multiplying the grades of occurrence assessment (O), severity assessment (S), and detection assessment (D). Although classical RPN is easy to use for its concise form, it is still criticized for its weaknesses. For example, traditional RPN does not take the weights of three risk factors into consideration. Besides, different combination of risk factors might acquire the same RPN, however, the potential risk might be totally different so that they might have different priorities. So far, many methods are presented. Nevertheless, most of them not only have rather complex algorithm, but also do not consider the non-exclusiveness between two rankings in assessment.

With the aim of solving these problems, in this paper, a novel method to risk evaluation based on D numbers theory is proposed. First of all, each assessment rating is treated as a fuzzy 
variable which is represented as a fuzzy number. Then, non-exclusive degree between two ratings can be calculated. Next, for each failure mode, the assessments of experts are aggregated by D numbers theory. The assessments are treated as D numbers and then are combined by using D numbers combination rule. Furthermore, RPNs are calculated for ranking the failure mode. Last but not the least, when coming to the same RPN of some failure modes, a variable, named risk coefficient, is defined to rank the failure modes with the same RPN. The large risk coefficient is, the more important degree it is supposed to be assigned, referring to the failure mode should be more priority to be corrected.

Step 1. Make sure the triangular fuzzy numbers for each rankings in assessment and calculate the non-exclusive degrees by 12 .

Step 2. The assessments of experts are regarded as D numbers, that is, for each failure mode, the assessments of each expert is constructed as a D number. Therefore, the aggregation of experts' assessment of each failure mode by combining the corresponding $\mathrm{D}$ numbers. If information is complete, the equations in Definition 8 are used to the combination. If the information is incomplete, the equations in Definition 10 are applied to the combination. What is more, if different experts have different weights, it is supposed to put the 17 into use.

Step 3. For the results of Step 2, use 5 to calculate pignistic probability transformation (PPT).

Step 4. Calculate RPN. Firstly, calculate the mathematical expectation of each assessment. Then, use Eq. (7) to calculate the RPN of each failure mode.

Step 5. Calculate risk coefficient.

Definition 12. Let $s$ be the standard deviation of the ratings of three assessments, defined as risk coefficient.

As mentioned above, different combination of risk factors might acquire the same RPN, however, the potential risk might be totally different. In this paper, risk coefficient, the standard deviation of the ratings of three assessments, is used to evaluate such kinds of failure modes. As a matter of fact, this method is reasonable. For example, there are two failure modes, the assessments of which are shown in Table 3.

Table 3: The assessments of three risk factors

\begin{tabular}{lccc}
\hline & occurrence assessment & severity assessment & detection assessment \\
\hline FM1 & 1 & 10 & 6 \\
FM2 & 2 & 6 & 5 \\
\hline
\end{tabular}

Apparently, two failure modes have the same RPN. However, as is shown in Table 3, compared with FM2, FM1 has the better grade in occurrence assessment but performs worse in severity and detection assessment. Therefore, FM1 should be more priority to be corrected. When observing and analyzing the data, it is not difficult to find that the distribution of FM2's data is more concentrated, which has smaller standard deviation. Besides, it is worth mentioning that standard deviation is usually used to measure the risk in financial field. Thus, it is reasonable that the standard deviation of the ratings of three assessments is applied to measure the risk in risk evaluation.

Step 6. Rank the failure modes though RPN. The larger RPN is, the more priority failure mode is supposed to be corrected. If the failure modes have the same RPN, their rankings depend on risk coefficient defined in Definition 12. The failure modes with the lager risk coefficient are assumed to be more significant and should be given higher priorities. 
Table 4: The results of the risk evaluation

\begin{tabular}{|c|c|c|c|c|c|c|c|c|c|}
\hline \multirow[t]{3}{*}{ Item } & \multicolumn{9}{|c|}{ Rating of risk factor } \\
\hline & \multicolumn{3}{|c|}{ Expert 1} & \multicolumn{3}{|c|}{ Expert 2} & \multicolumn{3}{|c|}{ Expert 3} \\
\hline & $\mathrm{O}$ & $\mathrm{S}$ & $\mathrm{D}$ & $\mathrm{O}$ & $\mathrm{S}$ & $\mathrm{D}$ & $\mathrm{O}$ & $\mathrm{S}$ & $\mathrm{D}$ \\
\hline 1 & $\begin{array}{l}3: 40 \% \\
4: 60 \%\end{array}$ & 7 & 2 & $\begin{array}{l}3: 90 \% \\
4: 10 \%\end{array}$ & 7 & 2 & $\begin{array}{l}3: 80 \% \\
4: 20 \%\end{array}$ & 7 & 2 \\
\hline 2 & 2 & 8 & 4 & 2 & $\begin{array}{l}8: 70 \% \\
9: 30 \%\end{array}$ & 4 & 2 & 8 & 4 \\
\hline 3 & 1 & 10 & 3 & 1 & 10 & 3 & 1 & 10 & 3 \\
\hline 4 & 1 & $\begin{array}{l}6: 80 \% \\
7: 20 \%\end{array}$ & 3 & 1 & 6 & $\begin{array}{l}3: 70 \% \\
2: 30 \%\end{array}$ & 1 & 6 & 3 \\
\hline 5 & 1 & 3 & $\begin{array}{l}2: 50 \% \\
1: 50 \%\end{array}$ & 1 & 3 & $\begin{array}{l}1: 70 \% \\
2: 30 \%\end{array}$ & 1 & $\begin{array}{l}3: 60 \% \\
2: 40 \%\end{array}$ & 1 \\
\hline 6 & 2 & 6 & 5 & 2 & 6 & 5 & 2 & 6 & 5 \\
\hline 7 & 1 & 7 & 3 & 1 & 7 & 3 & 1 & 7 & 3 \\
\hline 8 & 3 & $\begin{array}{l}5: 60 \% \\
6: 40 \%\end{array}$ & 1 & 3 & $\begin{array}{l}5: 80 \% \\
6: 20 \%\end{array}$ & 1 & 3 & $\begin{array}{l}5: 80 \% \\
7: 20 \%\end{array}$ & 1 \\
\hline 9 & $\begin{array}{l}2: 90 \% \\
1: 10 \%\end{array}$ & $\begin{array}{l}10: 60 \% \\
9: 40 \%\end{array}$ & 4 & $\begin{array}{l}2: 75 \% \\
1: 25 \%\end{array}$ & $\begin{array}{l}10: 90 \% \\
9: 10 \%\end{array}$ & 4 & $\begin{array}{l}2: 80 \% \\
1: 20 \%\end{array}$ & $\begin{array}{l}10: 90 \% \\
9: 10 \%\end{array}$ & 4 \\
\hline 10 & 1 & 10 & 6 & 1 & 10 & 6 & 1 & 10 & 6 \\
\hline 11 & 1 & 10 & 5 & 1 & 10 & 5 & 1 & 10 & 5 \\
\hline 12 & 1 & 10 & $\begin{array}{l}6: 60 \% \\
5: 40 \%\end{array}$ & 1 & 10 & $\begin{array}{l}5: 80 \% \\
4: 20 \%\end{array}$ & 1 & 10 & $\begin{array}{l}6: 70 \% \\
5: 30 \%\end{array}$ \\
\hline 13 & 1 & 10 & $\begin{array}{l}5: 80 \% \\
4: 20 \%\end{array}$ & 1 & 10 & 5 & 1 & 10 & 5 \\
\hline 14 & 1 & 10 & 6 & 1 & 10 & $\begin{array}{l}6: 80 \% \\
7: 20 \%\end{array}$ & 1 & 10 & 6 \\
\hline 15 & 2 & $\begin{array}{l}7: 95 \% \\
6: 5 \%\end{array}$ & 3 & 2 & 7 & 3 & 2 & 7 & $\begin{array}{l}3: 70 \% \\
4: 30 \%\end{array}$ \\
\hline 16 & $\begin{array}{l}2: 90 \% \\
1: 10 \%\end{array}$ & 4 & 3 & $\begin{array}{l}2: 75 \% \\
1: 25 \%\end{array}$ & 4 & 3 & $\begin{array}{l}2: 80 \% \\
1: 20 \%\end{array}$ & 4 & $\begin{array}{l}3: 80 \% \\
2: 20 \%\end{array}$ \\
\hline 17 & 2 & $\begin{array}{l}5: 90 \% \\
6: 10 \%\end{array}$ & 3 & 2 & $\begin{array}{l}5: 90 \% \\
6: 10 \%\end{array}$ & 3 & 2 & $\begin{array}{l}5: 60 \% \\
6: 40 \%\end{array}$ & 3 \\
\hline
\end{tabular}

\section{Numerical example}

In order to demonstrate the effectiveness and superiority of the proposed method, a numerical example in [77] is solved in this section. Supposing there are three experts who evaluate 17 failure modes and identify the ratings of the three risk factors. The assessment results are expressed in Table 4. In this illustrative example, the weights of experts and risk factors are supposed to be equal to 1 . Taking the failure mode 1 for example, the detailed computing is expressed as follows:

Step 1. The ratings are represented by triangular fuzzy numbers listed in Table 5 and shown in Figure 3. From Table 4, the occurrence assessments of three experts are $(3: 40 \%, 4: 60 \%)$, $(3: 90 \%, 4: 10 \%)$ and $(3: 80 \%, 4: 20 \%)$. Therefore, according to the Eq. (12), the non- 


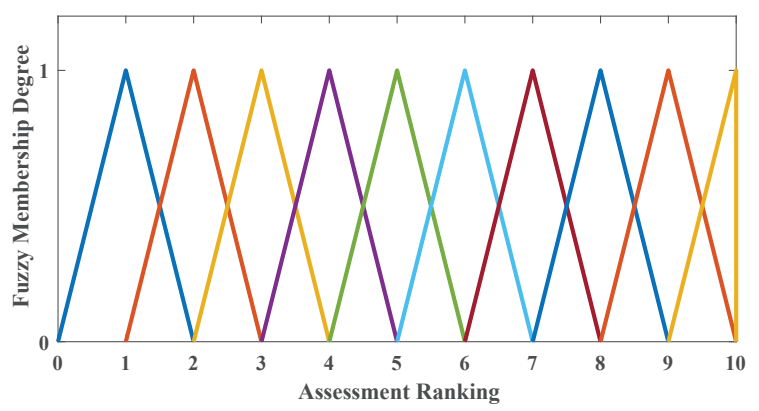

Figure 3: Graphically presentation of fuzzy variables in Table 5

Table 5: The fuzzy numbers of the assessment ratings

\begin{tabular}{cc}
\hline Assessment Ratings & Fuzzy Numbers \\
\hline 1 & $(0,1,2)$ \\
2 & $(1,2,3)$ \\
3 & $(2,3,4)$ \\
4 & $(3,4,5)$ \\
5 & $(4,5,6)$ \\
6 & $(5,6,7)$ \\
7 & $(6,7,8)$ \\
8 & $(7,8,9)$ \\
9 & $(8,9,10)$ \\
10 & $(9,10,10)$ \\
\hline
\end{tabular}

exclusiveness degrees are calculated as follows:

$$
\begin{gathered}
u_{\neg E}(3,3)=1, \quad u_{\neg E}(4,4)=1, \quad u_{\neg E}(3,\{3,4\})=1 \\
u_{\neg E}(4,\{3,4\})=1, \quad u_{\neg E}(3,4)=\frac{0.25}{1+1-0.25} \approx 0.1429
\end{gathered}
$$

Step 2. Construct D numbers and combine D numbers. According to Section 3, corresponding to the evaluations of three experts, three D numbers are constructed as follows:

$$
\begin{array}{llrl}
D_{1}(3) & =0.4, & & D_{1}(4)=0.6 \\
D_{2}(3)=0.9, & D_{2}(4)=0.1 \\
D_{3}(3)=0.8, & D_{3}(4)=0.2
\end{array}
$$

Then, because these $\mathrm{D}$ numbers have complete information, the combination among $D_{1}, D_{2}$ and $D_{3}$ is calculated by $10-14$, that is $D_{1} \odot D_{2} \odot D_{3}$.

At first, calculate $D^{\prime}=D_{1} \odot D_{2}$ :

$$
\begin{aligned}
K_{D^{\prime}} & =(1-0.1429) \times[0.4 \times 0.1+0.6 \times 0.9]=0.4971 \\
D^{\prime}(3) & =\frac{1}{1-0.4971} \times 1 \times 0.4 \times 0.9=0.7159 \\
D^{\prime}(4) & =\frac{1}{1-0.4971} \times 1 \times 0.6 \times 0.1=0.1193 \\
D^{\prime}(3,4) & =\frac{1}{1-0.4971} \times 0.1429 \times[0.4 \times 0.1+0.6 \times 0.9]=0.1648
\end{aligned}
$$




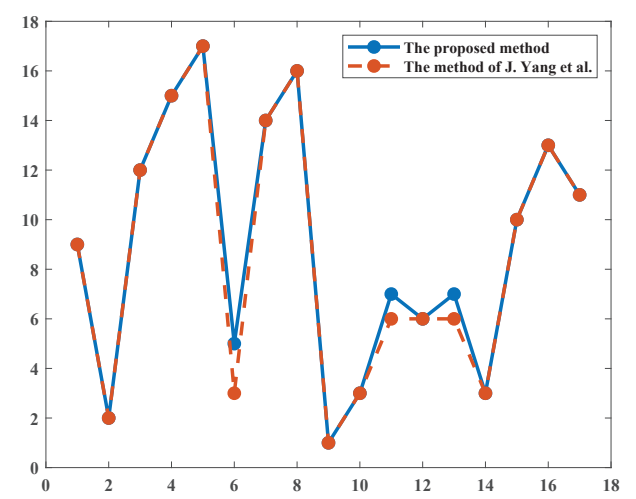

Figure 4: The risk priority of the failure modes using two methods

Next, calculate $D=D^{\prime} \odot D_{3}$ :

$$
\begin{aligned}
K_{D} & =(1-0.1429) \times[0.7159 \times 0.2+0.1193 \times 0.8]=0.2045 \\
D(3) & =\frac{1}{1-0.2045} \times[1 \times 0.7159 \times 0.8+1 \times 0.1648 \times 0.8]=0.8857 \\
D(4) & =\frac{1}{1-0.2045} \times[1 \times 0.1193 \times 0.2+1 \times 0.1648 \times 0.2]=0.0714 \\
D(3,4) & =\frac{1}{1-0.2045} \times 0.1429 \times[0.7159 \times 0.2+0.1193 \times 0.8]=0.0429
\end{aligned}
$$

Step 3. Calculate pignistic probability transformation (PPT) of the result of Step 2 by 5

$$
\operatorname{Bet} P(3)=0.8857+\frac{0.0429}{2} \approx 0.9071 \operatorname{Bet} P(4)=0.8857+\frac{0.0429}{2} \approx 0.0929
$$

Step 4. The mathematical expectation of the occurrence assessment is $3 \times 0.9071+4 \times$ $0.0929=3.0929$. Besides, three experts have the same evaluation to the severity assessment $(\mathrm{S})$ and detection assessment (D) of failure mode 1, what is more, the evaluation are real numbers, 7 and 2, without any uncertainty. Thus, in these two assessments, the results through Step 1 to 4 are still 7 and 2 .

Step 5. Calculate the RPN of failure mode $1\left(R P N_{1}\right)$ by 7 .

$$
R P N_{1}=O_{1} \times S_{1} \times D_{1}=3.0929 \times 7 \times 2=43.3006
$$

Step 6. Calculate the risk coefficient of the failure mode 1 .

$$
\begin{gathered}
\bar{x}_{1}=\frac{3.0929+7+2}{3} \approx 4.0310 \\
s_{1}=\sqrt{\frac{1}{3} \cdot\left[(3.0929-4.0310)^{2}+(7-4.0310)^{2}+(2-4.0310)^{2}\right]} \approx 2.6287
\end{gathered}
$$

The data of other failure modes are treated through Step 1 to 6 as mentioned above. Then, according to the RPNs and risk coefficients, the risk priorities of the failure modes are obtained, which are shown in Table 6. Meanwhile, in order to demonstrate the effectiveness of the proposed method, the results are compared with that of the J. Yang et al.'s method [77].

As shown in Table 6, the results of two methods are similar. Apart from failure mode 6, 11 and 13, other failure modes have the same risk priority rankings in both two methods. In addition, it is indicated that the five of highest risk priority rankings are failure mode $9,2,10$, 
Table 6: The results of the risk evaluation

\begin{tabular}{ccccc}
\hline Failure mode & RPN & Risk coefficient & $\begin{array}{c}\text { The rankings of } \\
\text { the proposed } \\
\text { method }\end{array}$ & $\begin{array}{c}\text { The rankings of } \\
\text { the J. Yang et al.'s } \\
\text { method }\end{array}$ \\
\hline 1 & 43.3006 & 2.6287 & 9 & 9 \\
2 & 64 & 3.0551 & 2 & 2 \\
3 & 30 & 4.7258 & 12 & 12 \\
4 & 18 & 2.5166 & 15 & 15 \\
5 & 3.0726 & 1.1478 & 17 & 17 \\
6 & 60 & 2.0817 & 5 & 3 \\
7 & 21 & 3.0551 & 14 & 14 \\
8 & 15.0657 & 2.0110 & 16 & 16 \\
9 & 78.10103376 & 4.1593 & 1 & 3 \\
10 & 60 & 4.5092 & 3 & 6 \\
11 & 50 & 4.5092 & 7 & 6 \\
12 & 51.452 & 4.5047 & 6 & 3 \\
13 & 50 & 4.5092 & 7 & 10 \\
14 & 60 & 4.5092 & 3 & 13 \\
15 & 42 & 2.6458 & 10 & 11 \\
16 & 23.5152 & 1.0203 & 13 & 11 \\
17 & 30.4038 & 1.5643 & 11 & \\
\hline
\end{tabular}

14, and 6, which refers that these 5 faults are most likely to occur. Furthermore, in both two methods, failure mode $16,7,4,8$, and 5 have the five of lowest priorities, indicating that these 5 failures are almost impossible to happen.

Figure 4 shows the comparison of risk priorities of two methods, in which the ranking is on the abscissa axis while the failure mode is on the vertical axis. As shown in Figure 4, two curves have the similar trend, which indicates that the proposed method is as effective as J. Yang et al.'s method.

Nevertheless, the results also reflect the different evaluations using two methods, which precisely demonstrates the superiority of the proposed method. As seen in Figure 4, using J. Yang et al.'s method, failure mode 11, 12, 13 have the same risk priority, while failure mode 12 has the larger risk priority compared with failure mode 11 and failure mode 13 in the proposed method. Because the highest rating of failure mode 12 in detection assessment is 6 , which is higher than that of failure mode 11, 13 in detection assessment. Thus, failure mode 12 is obviously supposed to have higher risk priority compare with failure mode 11 and failure mode 13 . What is more, in J. Yang et al.'s method, failure mode 6, 10, 14 have the same risk priority, but in the proposed method, failure mode 6 has the lower risk priority compared with failure mode 10 and failure mode 14. Although these failure modes have the same RPN, compared with failure mode 10 and failure mode 14, failure mode 6 has two lower risk ratings in severity assessment and detection assessment and merely a lager rating in occurrence assessment. Therefore, failure mode 6 is supposed to have the lower risk priority compared with failure mode 10 and failure mode 14 . 


\section{Conclusion}

In this paper, a novel method to risk evaluation in failure mode and effects analysis based on D numbers theory is proposed. In the proposed method, the application of the D numbers not only aggregates the fuzzy assessment in risk evaluation, but also takes the non-exclusiveness into account. Besides, the shortcomings of RPN are overcome successfully. Furthermore, the numerical example has demonstrated that the proposed method achieves less computation complexity compared with most existing method to risk evaluation in FMEA. In conclusion, the proposed method is an advanced and efficient method to risk evaluation in FMEA.

\section{Acknowledgement}

The work is partially supported by National Natural Science Foundation of China (Grant No. 61573290,61973332).

\section{Conflict of Interest}

The authors declare no conflict of interest.

\section{Bibliography}

[1] Adar, E.; Ince, M.; Karatop, B.; Bilgili, M. S. (2017). The risk analysis by failure mode and effect analysis (fmea) and fuzzy-fmea of supercritical water gasification system used in the sewage sludge treatment. Journal of Environmental Chemical Engineering, 5 (1), 1261-1268, 2017.

[2] Ahn, J.; Noh, Y.; Park, S. H.; Choi, B. I.; Chang, D.; Ahn, J.; Noh, Y.; Park, S. H.; Choi, B. I.; Chang, D. (2017). Fuzzy-based failure mode and effect analysis (fmea) of a hybrid molten carbonate fuel cell (mcfc) and gas turbine system for marine propulsion. Journal of Power Sources, 364, 226-233, 2017.

[3] Alimohammadzadeh, K.; Bahadori, M.; Jahangir, T.; Ravangard, R. (2017). Assessing common medical errors in a children's hospital nicu using failure mode and effects analysis (fmea). Trauma Monthly, in Press, 2017.

[4] Biswas, S. K.; Devi, D.; Chakraborty, M. (2018). A hybrid case based reasoning model for classification in internet of things (iot) environment. Journal of Organizational and End User Computing (JOEUC), 30 (4), 104-122, 2018.

[5] Cao, X.; Deng, Y. 2019. A new geometric mean fmea method based on information quality. IEEE Access, 7 (1), 95547-95554, 2019.

[6] Capuano, N.; Chiclana, F.; Herrera-Viedma, E.; Fujita, H.; Loia, V. (2018). Fuzzy rankings for preferences modeling in group decision making. International Journal of Intelligent Systems, 33 (7), 1555-1570, 2018.

[7] Chatterjee, K.; Zavadskas, E. K.; Tamosaitiene, J.; Adhikary, K.; Kar, S. (2018). A hybrid MCDM technique for risk management in construction projects. Symmetry, 10 (2), 46, 2018.

[8] Chen, Y. C.; Tsai, P. Y. (2017). Evaluating the operational risks of biomedical waste using failure mode and effects analysis. Waste Manag Res, 35 (6), 593-601, 2017. 
[9] Chin, K. S.; Wang, Y. M. ; Poon, G. K. K. et al. (2009). Failure mode and effects analysis using a group-based evidential reasoning approach[J]. Computers and Operations Research, 36 (6), 1768-1779, 2009.

[10] Dempster, A. P. (1967). Upper and lower probabilities induced by a multivalued mapping, Annals of Mathematical Statistics, 38 (2), 325-339, 1967.

[11] Deng, X.; Jiang, W. (2018). Dependence assessment in human reliability analysis using an evidential network approach extended by belief rules and uncertainty measures. Annals of Nuclear Energy, 117, 183-193, 2018.

[12] Deng, X.; Jiang, W. (2019). Evaluating green supply chain management practices under fuzzy environment: a novel method based on D number theory. International Journal of Fuzzy Systems, 21, 1389-1402, 2019.

[13] Deng, X.; Deng, Y. (2014). D numbers theory: a generalization of dempster-shafer theory. Computer Science, 2014.

[14] Dzitac, I.; Filip, F. G.; Manolescu, M.-J. (2017). Fuzzy logic is not fuzzy: World-renowned computer scientist Lotfi A. Zadeh. International Journal of Computers Communications \& Control, 12 (6), 748-789, 2017.

[15] Fang, R.; Liao, H.; Yang, J.-B.; Xu, D.-L. (2019). Generalised probabilistic linguistic evidential reasoning approach for multi-criteria decision-making under uncertainty. Journal of the Operational Research Society, in press, 2019.

[16] Fei, L.; Wang, H.; Chen, L.; Deng, Y. (2019). A new vector valued similarity measure for intuitionistic fuzzy sets based on owa operators. Iranian Journal of Fuzzy Systems, 16 (3), 113-126, 2019.

[17] Feng, F.; Fujita, H.; Ali, M. I.; Yager, R. R.; Liu, X. (2018). Another view on generalized intuitionistic fuzzy soft sets and related multiattribute decision making methods, IEEE Transactions on Fuzzy Systems, 27 (3), 474-488, 2018.

[18] Gao, X.; Deng, Y. (2019). The generalization negation of probability distribution and its application in target recognition based on sensor fusion. International Journal of Distributed Sensor Networks, 15 (5), 2019.

[19] Gao, X.; Deng, Y. (2019). The negation of basic probability assignment, IEEE Access, 7 (1), 107006-107014, 2019.

[20] Guan, X.; Liu, H.; Yi, X.; Zhao, J. (2018). The Improved Combination Rule of D Numbers and Its Application in Radiation Source Identification. Mathematical Problems in Engineering, 2018, 10 pages, 2018.

[21] Jiang, W.; Cao, Y.; Deng, X. (2019). A Novel Z-network Model Based on Bayesian Network and Z-number. IEEE Transactions on Fuzzy Systems, 2019.

[22] Jiang, W.; Wei, B. (2018). Intuitionistic fuzzy evidential power aggregation operator and its application in multiple criteria decision-making. International Journal of Systems Science, 49 (3), 582-594, 2018.

[23] Jiang, W.; Wei, B.; Liu, X.; Li, X.; Zheng, H. (2018). Intuitionistic fuzzy power aggregation operator based on entropy and its application in decision making, International Journal of Intelligent Systems, 33 (1), 49-67, 2018. 
[24] Jin, T.; Chen, C.; Chen, L.; Tian, H.; Zhu, D.; Jia, X. (2018). Failure mode and effects analysis of CNC machine tools based on spa, International Conference on System Reliability \& Safety, 107-111, 2018.

[25] Kang, B.; Deng, Y. (2019). The maximum Deng entropy, IEEE Access, 7 (1), 10.1109/ACCESS.2019.2937679, 2019.

[26] Kang, B.; Deng, Y.; Hewage, K.; Sadiq, R. (2019a). A method of measuring uncertainty for Z-number. IEEE Transactions on Fuzzy Systems, 27 (4), 731-738, 2019.

[27] Kang, B.; Zhang, P.; Gao, Z.; Chhipi-Shrestha, G.; Hewage, K.; Sadiq, R. (2019b). Environmental assessment under uncertainty using dempster-shafer theory and z-numbers. Journal of Ambient Intelligence and Humanized Computing, Published online, doi: 10.1007/s12652019-01228-y, 2019.

[28] Kim, K. O.; Zuo, M. J.; Kim, K. O.; Zuo, M. J. (2018). General model for the risk priority number in failure mode and effects analysis. Reliability Engineering \& System Safety, 169, 321-329, 2018.

[29] Lee, Y. C.; Kim, Y.; Huynh, J. W.; Hamilton, R. J. (2017). Failure modes and effects analysis for ocular brachytherapy. Brachytherapy, 16 (6), 1265-1279, 2017.

[30] Li, M.; Xu, H.; Deng, Y. (2019). Evidential decision tree based on belief entropy. Entropy, 21 (9), 897, 2019.

[31] Li, X.; Chen, X. (2018). D-Intuitionistic hesitant fuzzy sets and their application in multiple attribute decision making. Cognitive Computation, 10 (3), 496-505, 2018.

[32] Li, X.; He, M.; Wang, H. (2017). Application of failure mode and effect analysis in managing catheter-related blood stream infection in intensive care unit. Medicine, 96 (51), e9339, 2017.

[33] Li, Y.; Deng, Y. (2019). Intuitionistic Evidence Sets, IEEE Access, 7 (1), 106417-106426, 2019.

[34] Lin, S.; Li, C.; Xu, F.; Liu, D.; Liu, J. (2018). Risk identification and analysis for new energy power system in China based on D numbers and decision-making trial and evaluation laboratory (DEMATEL). Journal of Cleaner Production, 180, 81-96, 2018.

[35] Liu, B.; Hu, Y.; Deng Y. (2018). New Failure Mode and Effects Analysis based on D Numbers Downscaling Method, International Journal of Computers Communications \& Control, 13(2), 205-220, 2018.

[36] Liu, F.; Gao, X.; Zhao, J.; Deng, Y. (2019a). Generalized belief entropy and its application in identifying conflict evidence. IEEE Access 7 (1), 126625-126633, 2019.

[37] Liu, H.; Li, Z.; Song, W.; Su, Q. (2017). Failure mode and effect analysis using cloud model theory and PROMETHEE method. IEEE Trans. Reliability, 66 (4), 1058-1072, 2017.

[38] Liu, P.; Zhang, X. (2019). A multicriteria decision-making approach with linguistic D numbers based on the Choquet integral. Cognitive Computation, 11 (4), 560-575, 2019.

[39] Liu, Q.; Tian, Y.; Kang, B. (2019b). Derive knowledge of z-number from the perspective of dempster-shafer evidence theory. Engineering Applications of Artificial Intelligence, 85, 754-764, 2019. 
[40] Liu, Z.; Liu, Y.; Dezert, J.; Cuzzolin, F. (2019c). Evidence combination based on credal belief redistribution for pattern classification. IEEE Transactions on Fuzzy Systems, DOI: 10.1109/TFUZZ.2019.2911915, 2019.

[41] Liu, Z.; Pan, Q.; Dezert, J.; Han, J.-W.; He, Y. (2018a). Classifier fusion with contextual reliability evaluation. IEEE Transactions on Cybernetics, 48 (5), 1605-1618, 2018.

[42] Liu, Z.-G.; Pan, Q.; Dezert, J.; Martin, A. (2018b). Combination of classifiers with optimal weight based on evidential reasoning. IEEE Transactions on Fuzzy Systems, 26 (3), 12171230, 2018.

[43] Luo, C.; Chen, Y.; Xiang, H.; Wang, W.; Wang, Z. (2018). Evidence combination method in time domain based on reliability and importance. Journal of Systems Engineering and Electronics, 29 (6), 1308-1316, 2018.

[44] Luo, Z.; Deng, Y. (2019). A matrix method of basic belief assignment's negation in Dempster-Shafer theory. IEEE Transactions on Fuzzy Systems, 10.1109/TFUZZ.2019.2930027, 2019.

[45] Meng, D.; Li, Y.; Zhu, S.-P.; Lv, G.; Correia, J.; de Jesus, A. (2019). An enhanced reliability index method and its application in reliability-based collaborative design and optimization. Mathematical Problems in Engineering, 2019, 10 pages, 2019.

[46] Meng, D.; Liu, M.; Yang, S.; Zhang, H.; Ding, R. (2018). A fluid-structure analysis approach and its application in the uncertainty-based multidisciplinary design and optimization for blades. Advances in Mechanical Engineering, 10 (6), 2018.

[47] Meng, D.; Yang, S.; Zhang, Y.; Zhu, S.-P. (2019). Structural reliability analysis and uncertainties-based collaborative design and optimization of turbine blades using surrogate model. Fatigue 83 Fracture of Engineering Materials \& Structures, 42(6), 1219-1227, 2019.

[48] Mo, H.; Deng, Y. (2019). An evaluation for sustainable mobility extended by D numbers. Technological and Economic Development of Economy, 25 (5), 802-819, 2019.

[49] Moreno, R. V.; Riera, S. I.; Alvarez, E. M.; Mendoza, J. A. B.; Vazquez, S. T.; Castellano, L. D.; Gonzalez, J. C. M. (2016). Improvement of the safety of a clinical process using failure mode and effects analysis: Prevention of venous thromboembolic disease in critically ill patients. Medicina Intensiva, 40 (8), 483-490, 2016.

[50] Peeters, J. F. W.; Basten, R. J. I.; Tinga, T. (2018). Improving failure analysis efficiency by combining fta and fmea in a recursive manner. Reliability Engineering \& System Safety, 172, 36-44, 2018.

[51] Schuller, B. W.; Burns, A.; Ceilley, E. A.; King, A.; Letourneau, J.; Markovic, A.; Sterkel, L.; Taplin, B.; Wanner, J.; Albert, J. M. (2017). Failure mode and effects analysis: A community practice perspective, Journal of Applied Clinical Medical Physics, 18 (6), 258$267,2017$.

[52] Seiti, H.; Hafezalkotob, A. (2018). Developing pessimistic-optimistic risk-based methods for multi-sensor fusion: An interval-valued evidence theory approach. Applied Soft Computing, $72,609-623,2018$.

[53] Seiti, H.; Hafezalkotob, A.; MartĂnez, L. (2019). R-numbers, a new risk modeling associated with fuzzy numbers and its application to decision making, Information Sciences, 483, 206$231,2019$. 
[54] Seiti, H.; Hafezalkotob, A.; Najafi, S.; Khalaj, M. (2018). A risk-based fuzzy evidential framework for FMEA analysis under uncertainty: An interval-valued DS approach, Journal of Intelligent \& Fuzzy Systems, 35 (2), 1419-1430, 2018.

[55] Shafer, G. (1976). A Mathematical Theory of Evidence, Princeton University Press, Princeton, 1976.

[56] Shankar, R.; Choudhary, D.; Jharkharia, S. (2018). An integrated risk assessment model: A case of sustainable freight transportation systems, Transportation Research Part D: Transport and Environment, 63, 662-676, 2018.

[57] Smets, P.; Kennes, R. (1994). The transferable belief model. Artificial Intelligence, 66 (2), 191-234, 1994.

[58] Song, Y.; Deng, Y. (2019). A new method to measure the divergence in evidential sensor data fusion. International Journal of Distributed Sensor Networks, 15 (4), DOI: 10.1177/1550147719841295, 2019.

[59] Song, Y.; Deng, Y. (2019). Divergence measure of belief function and its application in data fusion. IEEE Access, 7 (1), 107465-107472, 2019.

[60] Stojkovic, T.; Marinkovic, V.; Jaehde, U.; Manser, T. (2016). Using failure mode and effects analysis to reduce patient safety risks related to the dispensing process in the community pharmacy setting. Research in Social \& Administrative Pharmacy Rsap, 13 (6), 1159-1166, 2016.

[61] Su, X.; Li, L.; Shi, F.; Qian, H. (2018). Research on the fusion of dependent evidence based on mutual information. IEEE Access 6, 71839-71845, 2018.

[62] Sun, L.; Liu, Y.; Zhang, B.; Shang, Y.; Yuan, H.; Ma, Z. (2016). An Integrated DecisionMaking Model for Transformer Condition Assessment Using Game Theory and Modified Evidence Combination Extended by D Numbers, Energies, 9 (9), 697, 2016.

[63] Sun, R.; Deng, Y. (2019a). A new method to identify incomplete frame of discernment in evidence theory. IEEE Access 7 (1), 15547-15555.

[64] Sun, R.; Deng, Y. (2019b). A new method to determine generalized basic probability assignment in the open world. IEEE Access, 7 (1), 52827-52835, 2019.

[65] Tooranloo, H. S.; Ayatollah, A. S. (2016). Pathology the internet banking service quality using failure mode and effect analysis in interval-valued intuitionistic fuzzy environment. International Journal of Fuzzy Systems. 19 (1), 1-15, 2016.

[66] Wang, N.; Liu, X.; Wei, D. (2018). A Modified D Numbers' Integration for Multiple Attributes Decision Making. IEEE Transactions on Fuzzy Systems, 20 (1), 104-115, 2018.

[67] Wang, N.; Wei, D. (2018). A Modified D Numbers Methodology for Environmental Impact Assessment. Technological and Economic Development of Economy 24 (2), 653-669, 2018.

[68] Wang, Y.; Zhang, K.; Deng, Y. (2019). Base belief function: an efficient method of conflict management. Journal of Ambient Intelligence and Humanized Computing, 10 (9), 3427-3437, 2019. 
[69] Wang, Z.; Gao, J.-M.; Wang, R.-X.; Chen, K.; Gao, Z.-Y.; Zheng, W. (2018). Failure mode and effects analysis by using the house of reliability-based rough vikor approach. IEEE Transactions on Reliability, 67 (1), 230-248, 2018.

[70] Xia, J.; Feng, Y.; Liu, L.; Liu, D.; Fei, L. (2019). On entropy function and reliability indicator for D numbers. Applied Intelligence, 49 (9), 3248-3266, 2019.

[71] Xiao, F. (2019). EFMCDM: Evidential fuzzy multicriteria decision making based on belief entropy. IEEE Transactions on Fuzzy Systems, DOI: 10.1109/TFUZZ.2019.2936368, 2019.

[72] Xiao, F.; Zhang, Z.; Abawajy, J. (2019). Workflow scheduling in distributed systems under fuzzy environment. Journal of Intelligent \& Fuzzy Systems, DOI: 10.3233/JIFS-190483, Pre-press, 1-11, 2019.

[73] Xu, X.; Xu, H.; Wen, C.; Li, J.; Hou, P.; Zhang, J. (2018a). A belief rule-based evidence updating method for industrial alarm system design. Control Engineering Practice, 81, 73 84, 2018.

[74] Xu, X.; Zheng, J.; Yang, J.-B.; Xu, D.-L.; Chen, Y.-W. (2017). Data classification using evidence reasoning rule, Knowledge-Based Systems, 116, 144-151, 2017.

[75] Xu, X.-B.; Ma, X.; Wen, C.-L.; Huang, D.-R.; Li, J.-N. (2018b). Self-tuning method of pid parameters based on belief rule base inference. Information Technology and Control, 47 (3), 551-563, 2018.

[76] Yager, R. R. (2012). On Z-valuations using Zadeh's Z-numbers. International Journal of Intelligent Systems, 27 (3), 259-278, 2012.

[77] Yang, J.; Huang, H. Z.; He, L. P.; Zhu, S. P.; Wen, D. (2011). Risk evaluation in failure mode and effects analysis of aircraft turbine rotor blades using Dempster-Shafer evidence theory under uncertainty, Engineering Failure Analysis, 18 (8), 2084-2092, 2011.

[78] Yazdi, M.; Daneshvar, S.; Setareh, H. (2017). An extension to fuzzy developed failure mode and effects analysis (fdfmea) application for aircraft landing system. Safety Science, 98, 113-123, 2017.

[79] Zadeh, L. A. (1965). Fuzzy sets, Information \& Control, 8 (3), 338-353, 1965.

[80] Zhang, J.; Zhong, D.; Zhao, M.; Yu, J.; Lv, F., (2019). An Optimization Model for Construction Stage and Zone Plans of Rockfill Dams Based on the Enhanced Whale Optimization Algorithm. Energies, 12 (3), 466, 2019.

[81] Zhang, W.; Deng, Y. (2019). Combining conflicting evidence using the DEMATEL method. Soft computing, 23 (17), 8207-8216, 2019.

[82] Zhao, J.; Deng, Y. (2019). Performer selection in Human Reliability analysis: D numbers approach. International Journal of Computers Communications \& Control, 14 (3), 437-452, 2019 .

[83] Zhou, M.; Liu, X.; Yang, J. (2017). Evidential reasoning approach for MADM based on incomplete interval value. Journal of Intelligent \& Fuzzy Systems 33 (6), 3707-3721, 2017.

[84] Zhou, M.; Liu, X.-B.; Chen, Y.-W.; Yang, J.-B. (2018). Evidential reasoning rule for MADM with both weights and reliabilities in group decision making. Knowledge-Based Systems, 143, 142-161, 2018. 
[85] Zhou, M.; Liu, X.-B.; Yang, J.-B.; Chen, Y.-W.; Wu, J. (2019). Evidential reasoning approach with multiple kinds of attributes and entropy-based weight assignment, KnowledgeBased Systems, 163, 358-375, 2019. 